Research Paper

\title{
Risk Factors for Long-term Outcome of Drug-eluting Stenting in Adults with Early-onset Coronary Artery Disease
}

\author{
Guipeng An ${ }^{1,2}$, Zhongqi Du1,2, Xiao Meng1,2, Tao Guo ${ }^{1,2}$, Guishuang $\mathrm{Li}^{1,2}$, Yuguo Chen ${ }^{1,2}, \mathrm{Jifu}_{\mathrm{Li}}{ }^{1,2}$, \\ Fengshuang An ${ }^{1,2}$, Yun Zhang ${ }^{1,2}$, Wenjing Li ${ }^{\circledR}$, Cheng Zhang ${ }^{1,2}$ \\ 1. The Key Laboratory of Cardiovascular Remodeling and Function Research, Chinese Ministry of Education and Chinese Ministry of \\ Health, Shandong University Qilu Hospital, Jinan, Shandong 250012, China; \\ 2. Department of cardiology, Shandong University Qilu Hospital, Jinan, Shandong 250012, China; \\ 3. Fine Arts School of Shandong University, Jinan, Shandong 250012, China.
}

$\square$ Corresponding authors: Cheng Zhang, M.D., Ph.D. Department of cardiology, Shandong University Qilu Hospital, No. 107 Wenhua Xi Road, Jinan, Shandong 250012, China E-mail: zhangc@sdu.edu.cn. Or Wenjing Li MA Fine Arts School of Shandong University, Jinan, Shandong 250012, China E-mail: tianya_79@163.com Tel:+8653182169484 Fax:+8653186169356.

(c) Ivyspring International Publisher. This is an open-access article distributed under the terms of the Creative Commons License (http://creativecommons.org/ licenses/by-nc-nd/3.0/). Reproduction is permitted for personal, noncommercial use, provided that the article is in whole, unmodified, and properly cited.

Received: 2014.0I.29; Accepted: 2014.04.2I; Published: 20I4.05.10

\begin{abstract}
Objective We lack data on the long-term outcome of drug-eluting stenting in patients with early-onset coronary artery disease (CAD). Here, we investigated the association of traditional risk factors and major adverse cardiovascular events (MACEs) after drug-eluting stenting in patients with CAD who were $<50$ years old. Methods We enrolled 437 consecutive CAD patients $<50$ years old who underwent drug-eluting stenting and 132 subjects who were age- and sex-matched and angiographically shown to be disease free as controls. MACEs were analyzed in CAD patients for a median of 24 months [interquartile range 14-34 months]. Results Male patients accounted for $90.4 \%$ of cases. As compared with controls, patients with early-onset CAD had higher body mass index and rates of smoking, family history of CAD, and diabetes and hypercholesterolemia. During the hospital stay, I patient died, and the incidence of MACEs was I.I\%. At the end of follow-up, the overall death rate was $0.7 \%$. MACEs were observed in 54 patients (I2.4\%). On Cox proportional hazard analyses, positive family history and diabetes were independent risk factors of MACEs (HR 2.6I, 95\% confidence interval I.29-4.00, $p=0.002$; and HR $2.48,95 \%$ confidence interval $0.86-3.14, p=0.004$, respectively). Conclusions Drug-eluting stenting is a reliable treatment for patients with early-onset CAD. Positive family history of CAD and diabetes are independent risk factors of adverse cardiovascular events in this subgroup of patients after drug-eluting stent implantation.
\end{abstract}

Key words: early onset coronary artery disease; drug-eluting stent; family history; diabetes; risk factor.

\section{Introduction}

Patients with early onset coronary artery disease (CAD) are a special subgroup of atherosclerotic patients and account for $4 \%$ to $10 \%$ of all CAD patients. Young and old CAD patients show different risk factor profiles, clinical presentation, coronary lesion distribution and prognosis [1]. However, the relatively small number of young CAD patients has hindered attempts to understand the factors associated with long-term outcome of this population.

Percutaneous coronary intervention $(\mathrm{PCI})$ has become an important strategy for treating CAD, even for high-risk lesions, such as three-vessel disease and 
left main coronary disease [2]. Recently, several clinical trials have shown the efficacy of PCI in early-onset CAD. PCI in young adults has shown excellent results on follow-up and is considered the treatment of choice [3-5].

Drug-eluting stents have been widely used in PCI because of their significant reduction in target vessel revascularization as compared with bare-metal stents [6]. However, few studies have examined the prognosis of drug-eluting stenting in relatively young CAD patients. In this study, we performed a study to examine risk factors associated with adverse cardiovascular events after drug-eluting stent implantation in patients with early-onset CAD.

\section{Methods}

\section{Protocols}

We selected consecutive patients $<50$ years old who underwent drug-eluting stent implantation from January 2008 to December 2011 in Qilu Hospital, Shandong University. Exclusion criteria were history of familial dyslipidemia, type I diabetes, or endstage renal disease. Age- and gender-matched subjects angiographically proven to be disease free were selected as normal controls. The study was approved by the local review board and ethics committee, and all patients gave their written informed consent. Both patients and controls came from the same geographical area and had similar socioeconomic and ethnic backgrounds.

A family history of CAD was defined as the presence of at least one-first degree relative with CAD developing before age 55 years for men and 65 years for women. The body mass index (BMI) was calculated as weight $(\mathrm{kg})$ divided by height (meter) squared. Diabetes was defined as a fasting serum glucose level $>126 \mathrm{mg} / \mathrm{dl}$ or current use of any diabetes medication. Hypertension was defined as seated diastolic blood pressure $>90 \mathrm{mmHg}$, systolic blood pressure $>140 \mathrm{mmHg}$, or use of anti-hypertensive drugs. Hypercholesterolemia was defined as total cholesterol $>5.2 \mathrm{mmol} / \mathrm{L}$. Current smokers or drinkers were defined by self-reported current smoking or regular drinking.

Coronary angiography involved the Judkin technique with a quantitative coronary angiographic system. CAD extent was assessed in 2 ways as previously described[7]: 1) number of major coronary vessels with $>70 \%$ occlusion (left anterior descending, left circumflex, and right coronary arteries), namely, single-, double-, or triple-vessel disease, classified as grades 1-3 (> 50\% lesion in the left main coronary artery was considered as two-vessel disease); and 2) number of narrowed major coronary vessels (left main, left anterior descending, left circumflex, and right coronary arteries) and/or their second-order branches (up to 2 large diagonals, 2 large marginals, and the posterior descending artery) with any narrowing, classified as grades 0-9. Coronary angiograms were interpreted by 2 investigators blinded to patient data.

\section{Study endpoints and definitions}

The follow-up information regarding health status and any hospital admissions was obtained from hospital records and by telephone in June 2012. The primary endpoint was the occurrence of major adverse cardiovascular events (MACEs), including death (noncardiacvascular death and cardiac death), myocardial infarction, stroke, and any revascularization (PCI or coronary artery bypass grafting). Cardiovascular death was defined as death due to myocardial infarction, stroke or sudden death without any obvious reasons. Definition of myocardial infarction was based on the third universal definition [8]. Target lesion revascularization was defined as any attempted percutaneous or surgical revascularization of lesions in the coronary artery after the initial procedure. Outcomes were assessed by 2 independent observers who were blinded to patient baseline, clinical, and laboratory data.

\section{Statistical analysis}

Analysis of normality of continuous variables involved the Kolmogorov-Smirnov test. Normally distributed continuous variables are described by mean $\pm S D$. Non-normally distributed variables are described by median (interquartile range). Categorical data are described by number (percentage). Differences among groups were analyzed by $t$ test or Mann-Whitney $U$ test for continuous variables and chi-square or Fisher's exact test for categorical data. Cox proportional-hazards multiple regression analysis involved potential confounders associated with clinical outcome with $p$-value $<0.2$ included in the model. Two-sided $\mathrm{p}<0.05$ was considered statistically significant. All analyses involved use of SPSS 17.0 for Windows (SPSS, Inc., Chicago, IL, USA).

\section{Results}

From January 2008 to December 2011, 3625 patients had underwent drug-eluting stent implantation in Qilu Hospital, Shandong University. We finally recruited 437 patients with $>70 \%$ stenosis in at least 1 coronary vessel on angiography as early-onset CAD patients; the patients had been implanted with drug-eluting stents. We recruited 132 age- and gender-matched subjects angiographically proven to be disease free as normal controls. The prevalence of risk 
factors for cases and controls is in Table 1. Similar to previous reports $[9,10]$, most cases were male $(90.3 \%)$. As compared with controls, cases had higher BMI and rate of smoking, family history of $\mathrm{CAD}$, diabetes and hypercholesterolemia $(\mathrm{p}<0.05)$. Smoking was the most prevalent risk factor. In contrast, the groups did not differ in history of hypertension or rate of drinking $(p>0.05)$. The average number of risk factors (including smoking, family history, hypercholesterolemia, hypertension and diabetes) was $2.00 \pm 0.05$ for cases and $1.39 \pm 0.09$ for controls $(p<0.001)$.

Table I. Distribution of risk factors of coronary artery disease (CAD) among patients < 50 years old (early-onset CAD) who underwent drug-eluting stenting and controls.

\begin{tabular}{|c|c|c|c|}
\hline & $\begin{array}{l}\text { Control } \\
(n=132)\end{array}$ & $\begin{array}{l}\text { Patients } \\
(\mathrm{n}=437)\end{array}$ & $P$ value \\
\hline Age, years (no., range) & $45(40-47)$ & $44(41-47)$ & ns \\
\hline Men (no., \%) & $119(90.2)$ & 395(90.4) & ns \\
\hline Smoking (no., \%) & $61(46.2)$ & $288(65.9)$ & 0.00 \\
\hline Drinking (no., \%) & $79(59.9)$ & $250(57.2)$ & 0.59 \\
\hline BMI $\left(\mathrm{kg} / \mathrm{m}^{2} ;\right.$ no., range $)$ & $\begin{array}{l}25.21 \\
(22.79-27.76)\end{array}$ & $\begin{array}{l}26.62 \\
(24.22-29.3)\end{array}$ & 0.02 \\
\hline Family history of CAD (no., \%) & $20(15.2)$ & $128(29.3)$ & 0.00 \\
\hline Hypertension (no., \%) & $60(45.5)$ & $208(47.6)$ & 0.67 \\
\hline Diabetes (no., \%) & $15(11.4)$ & $111(25.4)$ & 0.00 \\
\hline Hypercholesterolemia (no., \%) & $29(22.0)$ & $137(31.4)$ & 0.04 \\
\hline
\end{tabular}

BMI, body mass index; ns=not significant.

The clinical characteristics of CHD patients are in Table 2. The most common clinical presentation was unstable angina (55.8\%), followed by ST-segment elevation MI (39.4\%) and non-ST-segment elevation MI $(4.8 \%)$. One patient had a previous diagnosis of renal failure and 2 patients had heart failure. Five patients $(1.1 \%)$ had a previous diagnosis of autoimmune or rheumatic diseases; 21 had a history of myocardial infarction and 6 of these had undergone PCI. The mean left ventricular ejection fraction was $0.59 \pm 0.11$.

Table 2. Clinical characteristics of patients with early-onset CAD.

\begin{tabular}{ll}
\hline Characteristic & No. of patients \\
\hline Clinical presentation & 244 \\
Unstable angina & 21 \\
Non-ST-segment elevation myocardial infarction & 172 \\
ST-segment elevation myocardial infarction & 1 \\
Renal failure & 2 \\
Chronic heart failure & 0 \\
Cocaine use & 0 \\
Use of contraceptive drugs & 5 \\
Autoimmune disease & 21 \\
Previous acute myocardial infarction & 0 \\
Previous coronary artery bypass grafting & 6 \\
Previous percutaneous coronary intervention & $0.59 \pm 0.11$ \\
Left ventricular ejection fraction (\%) & \\
\hline
\end{tabular}

Single-vessel disease was present in $42.1 \%$ of all cases, followed by double-vessel disease $(29.8 \%$, Table $3)$. Bifurcation lesions were found in 59 patients $(13.5 \%)$. In all, 314 patients had 1 to 2 stents implanted, 108 had 3 to 4 , and 15 had more than 4 . The mean stent length for cases was $50.21 \pm 1.50 \mathrm{~mm}$. Glycoprotein $\mathrm{IIb} / \mathrm{III}$ inhibitors were used in $27.7 \%$ of patients undergoing PCI. At discharge, almost all patients were prescribed aspirin, clopidogrel and statins $(91.2 \%)$. $\beta$-blockers, angiotensin-converting enzyme inhibitors, and angiotensin receptor blockers were prescribed for $81.3 \%, 39.2 \%$, and $23.3 \%$ of patients, respectively.

Table 3. Angiographic and procedural characteristics for patients with early-onset CAD.

\begin{tabular}{ll}
\hline Characteristic & No. of patients \\
\hline Diseased coronary artery & 7 \\
Left main & 184 \\
Single vessel & 130 \\
Double vessels & 123 \\
Triple vessels & 59 \\
Bifurcations & \\
Stent number per patient & 314 \\
$1-2$ & 108 \\
$3-4$ & 15 \\
$>4$ & $50.21 \pm 1.50$ \\
Mean drug-eluting stent length per patient (mm) & \\
Medications at discharge & $91.2 \%$ \\
Statins & $81.3 \%$ \\
$\beta$-blockers & $39.2 \%$ \\
Angiotensin-converting enzyme inhibitors & $23.3 \%$ \\
Angiotensin receptor blockers & $100 \%$ \\
Aspirin & $100 \%$ \\
Clopidogrel & $27.7 \%$ \\
Glycoprotein IIb/IIIa inhibitors &
\end{tabular}

No patient was lost to follow-up. The incidence of in-hospital and long-term adverse events are in Table 4. During the hospital stay, 5 patients experienced myocardial infarction. 1 patient died and 4 other patients underwent repeat PCI. No stroke was recorded. The incidence of in-hospital MACEs was $1.1 \%$. During a median follow-up of 24 months (interquartile range 14-34 months), the overall mortality was $0.7 \%$ (2 patients died from cardiac causes and 1 patient from liver cancer), and the incidence of myocardial infarction was $3.9 \%$. Repeat PCI was needed in $9.4 \%$ of patients $(n=41)$ and coronary artery bypass grafting in $0.5 \%(n=2)$. The incidence of all MACEs was $12.4 \%$. On Cox proportional-hazards multivariate analysis, family history of CAD and diabetes were independent predictors of MACEs with drug-eluting stenting for CAD (HR 2.61, 95\% confidence interval 1.29-4.00, $\mathrm{p}=0.002$; and HR 2.48, 95\% confidence in- 
terval 0.86-3.14, $\mathrm{p}=0.004$, respectively; Table 5), after adjusting for age, gender, CAD extent, smoking status, BMI, hypertension and hypercholesterolemia at baseline.

Table 4. Incidence of major adverse cardiovascular events among patients with early-onset CAD

\begin{tabular}{ll}
\hline Variable & No. $(\%)$ \\
\hline In-hospital events & $1(0.2)$ \\
Death & $5(1.1)$ \\
Myocardial infarction & 0 \\
Cerebrovascular accident & $4(0.9)$ \\
Repeat percutaneous coronary intervention & 0 \\
Coronary artery bypass grafting & \\
Events at long-term follow-up & $3(0.7)$ \\
Death & $17(3.9)$ \\
Myocardial infarction & $1(0.2)$ \\
Cerebrovascular accident & $25(5.7)$ \\
In-stent restenosis & $41(9.4)$ \\
Repeat percutaneous coronary intervention & $2(0.5)$ \\
Coronary artery bypass grafting & \\
\hline
\end{tabular}

Table 5. Cox proportional-hazards multivariate analysis of independent predictors of major adverse cardiovascular events

\begin{tabular}{llll}
\hline Tested Variable & $\mathrm{p}$ value & Hazard ratio & $95 \% \mathrm{CI}$ \\
\hline Family history & 0.002 & 2.61 & $1.29-4.00$ \\
Diabetes & 0.004 & 2.48 & $0.86-3.14$ \\
Dyshypercholesterolemia & 0.12 & 0.57 & $0.27-1.15$ \\
\hline
\end{tabular}

\section{Discussion}

Early-onset CAD requires special clinical attention because of its devastating impact on patients and society. Recently, PCI has shown encouraging results in relatively young people with CAD $[4,5]$, but data on drug-eluting stenting in this subset are limited. Most available published studies were conducted in the era before such stenting or examined both drug-eluting and bare-metal stenting. In this study, we performed long-term follow-up (median 24 months) of relatively young patients ( $<50$ years old) with $\mathrm{CAD}$ and revealed that drug-eluting stenting was a reliable treatment for early-onset CAD. Family history of CAD and diabetes were independent risk factors of adverse cardiovascular events in early-onset CAD with stent implantation.

Drug-eluting stenting is gaining in popularity among cardiologists because of its effectiveness in reducing restenosis rate. PCI seems to provide excellent results in the hospital and during long-term follow-up [11, 12]. Recently, drug-eluting stenting showed good short-term follow-up results in 26 patients with premature $\mathrm{CAD}$, with no target lesion re- vascularization [3]. In our study, after a median follow-up of 24 months (interquartile range 14-34 months), the overall mortality was $0.7 \%$ and the incidence of total MACEs was $12.4 \%$. These rates are relative lower than those in studies of ordinary populations $[13,14]$. Thus, drug-eluting stenting could be a good choice for therapy for early-onset CAD.

Family history of CAD is an important risk factor of early-onset CAD [1, 15], but little has been learned about the relationship between family history and prognosis for this subgroup of patients. The results of our study demonstrate that family history of CAD is an important predictor of major adverse cardiovascular events in relatively young CAD patients with drug-eluting stents. The influence of family history is independent of other well-known risk factors. Genetic background may play an important role in the development of CAD and may be associated with long-term outcomes in relatively young CAD patients with drug-eluting stenting, as was shown in unrestricted populations $[16,17]$.

Diabetes has been considered a risk factor of both early- and late-onset CAD [18]. Recently, diabetes was found an independent predictor of long-term mortality in premature CAD with PCI [4], but studies revealing the relationship between diabetes and outcomes of drug-eluting stenting in early onset CAD are lacking. In our study, after a median follow-up of 24 months, diabetes was found a strong risk factor of major cardiovascular events after drug-eluting stenting, which indicates the importance of diabetes control.

Recently, several large-scale studies have shown a strong association of traditional risk factors (such as male sex, family history, diabetes, dyslipidemia, and smoking) and early-onset CAD [19, 20]. Our study confirmed those previous finding by showing a significantly higher rate of smoking, family history of $\mathrm{CAD}$, diabetes, and hypercholesterolemia in relatively young patients with CAD. The average number of risk factors was also significantly greater in patients than controls, which implies the importance of traditional risk factors in the development of early-onset CAD. Gender and smoking should be monitored because of a high difference between patients and controls in these factors.

Our study contains some limitations. Firstly, the majority of end points are repeat revascularization. A much larger sample size would be needed to have necessary power for a more robust Death/MI endpoint. Secondly, although we showed the overt association of family history of CAD and diabetes with long-term outcomes of drug-eluting stenting, the sample was from a single center, so results may not be representative of the general population. Thirdly, the 
population was small. The conclusion needs to be examined in a larger population and a better-designed study.

In conclusion, our study showed that drug-eluting stenting is a reliable treatment for Chinese patients with early onset CAD. Family history of $\mathrm{CAD}$ and diabetes are independent risk factors of adverse cardiovascular events in early onset CAD with such stenting.

\section{Abbreviations}

CAD: coronary artery disease; MACE: major adverse cardiovascular events; PCI: percutaneous coronary intervention; BMI: body mass index.

\section{Acknowledgements}

This study was supported by the National Natural Science Foundation of China (No. 81000125 and 81000127), Specialized Research Fund for the Doctoral Program of Higher Education (20100131120057) and Promotive Research Fund for Young and Middle-aged Scientists of Shandong Province (BS2012YY017).

\section{Competing Interests}

The authors have declared that no competing interest exists.

\section{References}

1. Doughty M, Mehta R, Bruckman D, Das S, Karavite D, Tsai T, et al. Acute myocardial infarction in the young--The University of Michigan experience. American heart journal. 2002; 143: 56-62.

2. Mohr FW, Morice MC, Kappetein AP, Feldman TE, Stahle E, Colombo A, et al. Coronary artery bypass graft surgery versus percutaneous coronary intervention in patients with three-vessel disease and left main coronary disease: 5-year follow-up of the randomised, clinical SYNTAX trial. Lancet. 2013; 381: 629-38.

3. Wang MH, Lee WL, Wang KY, Hsieh YC, Liu TJ, Lin IH, et al. Short-term follow-up results of drug-eluting stenting in premature coronary artery disease patients with multiple atherosclerotic risk factors. Journal of the Chinese Medical Association : JCMA. 2008; 71: 342-6.

4. Khawaja FJ, Rihal CS, Lennon RJ, Holmes DR, Prasad A. Temporal trends (over 30 years), clinical characteristics, outcomes, and gender in patients $</=50$ years of age having percutaneous coronary intervention. The American journal of cardiology. 2011; 107: 668-74.

5. Meliga E, et al. Long-term outcomes of percutaneous coronary interventions with stent implantation in patients $</=40$ years old. The American journal of cardiology. 2012; 109: 1717-21.

6. Kirtane AJ, Gupta A, Iyengar S, Moses JW, Leon MB, Applegate R, et al. Safety and efficacy of drug-eluting and bare metal stents: comprehensive meta-analysis of randomized trials and observational studies. Circulation. 2009: 119: 3198-206.

7. Pollak A, Rokach A, Blumenfeld A, Rosen LJ, Resnik L, Dresner Pollak R. Association of oestrogen receptor alpha gene polymorphism with the angiographic extent of coronary artery disease. European heart journal. 2004; 25: 240-5.

8. Thygesen K, Alpert JS, Jaffe AS, Simoons ML, Chaitman BR, White HD, et al. Third universal definition of myocardial infarction. Circulation. 2012; 126: 2020-35

9. Pineda J, Marin F, Roldan V, Valencia J, Marco P, Sogorb F. Premature myocardial infarction: clinical profile and angiographic findings. International journal of cardiology. 2008; 126 : 127-9.

10. Guella I, Rimoldi V, Asselta R, Ardissino D, Francolini M, Martinelli N, et al. Association and functional analyses of MEF2A as a susceptibility gene for premature myocardial infarction and coronary artery disease. Circulation Cardiovascular genetics. 2009; 2: 165-72.

11. De Luca G, Dirksen MT, Spaulding C, Kelbaek H, Schalij M, Thuesen L, et al. Meta-Analysis Comparing Efficacy and Safety of First Generation Drug-Eluting Stents to Bare-Metal Stents in Patients With Diabetes Mellitus
Undergoing Primary Percutaneous Coronary Intervention. The American journal of cardiology. 2013;111:1295-304

12. Bangalore S, Kumar S, Fusaro M, Amoroso N, Attubato MJ, Feit F, et al. Shortand long-term outcomes with drug-eluting and bare-metal coronary stents: a mixed-treatment comparison analysis of 117762 patient-years of follow-up from randomized trials. Circulation. 2012; 125: 2873-91.

13. Wykrzykowska J, Serruys P, Buszman P, Linke A, Ischinger T, Klauss V, et al. The three year follow-up of the randomised "all-comers" trial of a biodegradable polymer biolimus-eluting stent versus permanent polymer sirolimus-eluting stent (LEADERS). EuroIntervention : journal of EuroPCR in collaboration with the Working Group on Interventional Cardiology of the European Society of Cardiology. 2011; 7: 789-95.

14. Silber S, Windecker S, Vranckx P, Serruys PW, investigators RAC. Unrestricted randomised use of two new generation drug-eluting coronary stents: 2-year patient-related versus stent-related outcomes from the RESOLUTE All Comers trial. Lancet. 2011; 377: 1241-7.

15. Wiesbauer F, Blessberger H, Azar D, Goliasch G, Wagner O, Gerhold L, et al. Familial-combined hyperlipidaemia in very young myocardial infarction survivors (< or $=40$ years of age). European heart journal. 2009; 30: 1073-9.

16. Bachmann JM, Willis BL, Ayers CR, Khera A, Berry JD. Association between family history and coronary heart disease death across long-term follow-up in men: the Cooper Center Longitudinal Study. Circulation. 2012; 125: 3092-8.

17. Mulders TA, Maurissen LF, Meyer Z, Hameeteman M, van der Donk C, Kroon $\mathrm{AA}$, et al. A positive family history for premature cardiovascular disease identifies patients prone to recurrent arterial thrombotic events. European journal of preventive cardiology. 2012; 19: 1465-73.

18. Sadeghian S, Graili P, Salarifar M, Karimi AA, Darvish S, Abbasi SH. Opium consumption in men and diabetes mellitus in women are the most important risk factors of premature coronary artery disease in Iran. International journal of cardiology. 2010; 141: 116-8.

19. Zimmerman $\mathrm{FH}$, Cameron A, Fisher $\mathrm{LD}, \mathrm{Ng} \mathrm{G}$. Myocardial infarction in young adults: angiographic characterization, risk factors and prognosis (Coronary Artery Surgery Study Registry). Journal of the American College of Cardiology. 1995; 26: 654-61.

20. Gottlieb S, Boyko V, Zahger D, Balkin J, Hod H, Pelled B, et al. Smoking and prognosis after acute myocardial infarction in the thrombolytic era (Israeli Thrombolytic National Survey). Journal of the American College of Cardiology. 1996; 28: 1506-13. 